\title{
PHYMATOSORUS GROSSUS (POLYPODIACEAE) EN MÉXICO Y COMENTARIOS SOBRE OTROS PTERIDOBIONTES NO-NATIVOS
}

\author{
J. Daniel Tejero-Díez ${ }^{1,2}$ y Alin N. Torres-Díaz ${ }^{1}$ \\ ${ }^{1}$ Universidad Nacional Autónoma de México, Facultad de Estudios Superiores \\ Iztacala, Carrera de Biología, Avenida de los Barrios 1, Los Reyes Iztacala, \\ Tlalnepantla, 54090 Estado de México, México. \\ ²Autor para la correspondencia: tejero@unam.mx
}

\section{RESUMEN}

Se reporta el hallazgo de Phymatosorus grossus (Langsd. \& Fisch.) Brownlie en México; helecho exótico que presenta cualidades para comportarse como invasor. Se describe su morfología, características ecológicas y distribución. Además, se incluye una lista de otros licopodios y helechos no-nativos registrados en la bibliografía, así como de otras especies de pteridobiontes que probablemente han ingresado al país en tiempos recientes.

Palabras clave: helechos exóticos, helechos invasores, malezas, México, Phymatosorus.

\begin{abstract}
Phymatosorus grossus (Langsd. \& Fisch.) Brownlie is reported for Mexico for the first time. It is an exotic fern that presents characteristics typical of invasive species. We give a morphological description, ecological features and distribution of the species. We also include a list of other non-native clubmosses and ferns reported in the literature, as well as other species of pteridophytes that have probably arrived in Mexico recently.
\end{abstract}

Key words: alien ferns, invader ferns, Mexico, Phymatosorus, weeds.

Con el calificativo de plantas "no-nativas" se conoce a las especies exóticas o no-indígenas de una región dada, las cuales han sido introducidas en forma intencional o accidental debido a las actividades humanas (Pysek et al., 2004). 
El aprovechamiento que el ser humano ha hecho de las especies y del ambiente se ha traducido en un traspaso de plantas (y animales) entre localidades en todo el mundo. En ocasiones este proceso ocurre en forma consciente y en otras, las diásporas se trasladan como acompañantes ocultas (polizontes) o en forma fortuita aprovechándose de la antropización de los ambientes. Las especies no-nativas, categorizadas como naturalizadas, tienen la capacidad de un crecimiento independiente y sustentan un auto reemplazamiento de su población por al menos 10 años (Williamson, 1996). De éstas, sólo alrededor de 10\% se convierten en invasoras en el hábitat hospedante (Groves, 1991; Williamson y Fitter, 1996).

Las plantas invasoras son aquellas que producen diásporas, en ocasiones de una forma muy rápida o en gran número y que tienen el potencial de esparcirse sobre grandes áreas y causar un daño a ecosistemas naturales (Pysek et al., 2004). La invasión de comunidades naturales por la introducción de especies exóticas constituye una de las amenazas para la biodiversidad a nivel mundial (Adair y Groves, 1998; Baskin, 2002; Lodge, 1993). Se ha encontrado que las plantas invasoras pueden afectar el nicho de las especies nativas de una comunidad, alterar la estructura de un ecosistema e interrumpir los procesos evolutivos (D’Antonio y Vitousek, 1992; Mack et al., 2000 y Levine et al., 2003); tienen, por tanto, un efecto negativo en diversos hábitats en todo el mundo al competir y reducir la riqueza de especies nativas, alterar el régimen del agua o el fuego, afectar los procesos de los nutrientes del suelo y alterar la geomorfología en general (MacDonald et al., 1989; Cronk y Fuller, 1995; Rose y Fairweather, 1997).

La dispersión antrópica es favorecida por la industria hortícola de plantas ornamentales o por la inconsciencia de personas que pasan a escondidas propágulos para fines personales (Reichard y White, 2001; Mack y Erneberg, 2002). Se calcula que en Estados Unidos de América cerca de 5000 especies no-nativas han escapado del cultivo (Morse et al., 1995) y que las plantas invasoras causan un daño calculado en 34 mil millones de dólares (Pimentel, 2005). En México, Villaseñor y EspinosaGarcía (2004) listan 618 especies de plantas fanerógamas no-nativas, pero no se ha calculado cuántas son invasoras y el daño económico que ellas provocan. Mickel y Smith (2004) mencionan la presencia de nueve especies de helechos exóticos en México y, recientemente, Mora-Olivo y Yatskievych (2009) documentan la presencia de Salvinia molesta en el norte del país.

Durante los recorridos de campo del primer autor, desde el año 2000 a la fecha, en México y Centroamérica, se han obtenido ejemplares de dos poblaciones de una planta cuyos caracteres no coincidían con los de las especies reportadas para el país. Estos especímenes se determinaron con la ayuda de las claves de Stuart (2009) 
y la flora de Australia (Bostock y Spokes, 1998). El proceso de determinación dio como resultado el nombre de Phymatosorus grossus (Langsd. \& Fisch.) Brownlie. El ejemplar fue corroborado en línea con el isotipo en BM y con el herbario KALA (Kalaupapa Historic National Park Herbarium, Hawaii, USA, número KALA13288). Se realizó una búsqueda en la literatura y en los herbarios ENCB, FCMEX, MEXU, MO, NY, UC y UAMIZ para ubicar otras posibles poblaciones. Dada la ausencia de registros para México, se establece que Phymatosorus grossus ha sido recientemente introducido al país como planta de ornato y que estas poblaciones comienzan a escaparse a la vida silvestre.

Considerando que se trata de un género hasta ahora no incluido en las obras que tratan sobre los licopodios y helechos de México (y América continental), se incluye su descripción taxonómica:

Phymatosorus Pic. Serm., Webbia 28: 457 (1973). TIPO: Phymatosorus scolopendria (Burm.f.) Pic. Serm.

Helechos epífitos a epipétricos y ocasionalmente terrestres humícolas. Rizoma grueso, larga a cortamente rastrero, con escamas esparcidas, peltadas y clatradas (al menos en el centro). Hojas que nacen a intervalos amplios a lo largo del rizoma, pecíolo articulado a un filopodio; lámina simple y entera, lobada o profundamente pinnatífida, glabra; venas de tercer y cuarto orden anastomosadas y vénulas internas libres. Soros redondos o algo alargados, en 1(o 2) filas regulares a irregulares en cada lado de la vena primaria (costa) y/o secundarios, nacen en una concavidad de la lámina y se imprimen en la superficie adaxial formando una protuberancia de forma areolar. Esporas lisas a tuberculadas (Tindale, 1994).

Género con aproximadamente 12 especies nativas de África, Polinesia, Asia, Australia y Nueva Zelanda, principalmente en clima cálido.

Phymatosorus (sensu Bosman, 1991) ha sido reconocido en clasificaciones recientes de los helechos (Smith et al., 2008). Sin embargo, en diferentes floras se sigue considerando como parte de Microsorum, principalmente por la presencia de híbridos intergenéricos (Bostock y Spokes, 1998). La especie existente en el Neotrópico es:

Phymatosorus grossus (Langsd. \& Fisch.) Brownlie, Nova Hedwigia 55: 385 (1977) Fig. 1.

Polypodium grossum Langsd. \& Fisch., Pl. Voy. Russes Monde (Icon. Fil.) 1: 9, t. 8. 1810. HOLOTIPO: Langsdorff \& Fischer 1810. Nukahiva, Islas Marquesas, 

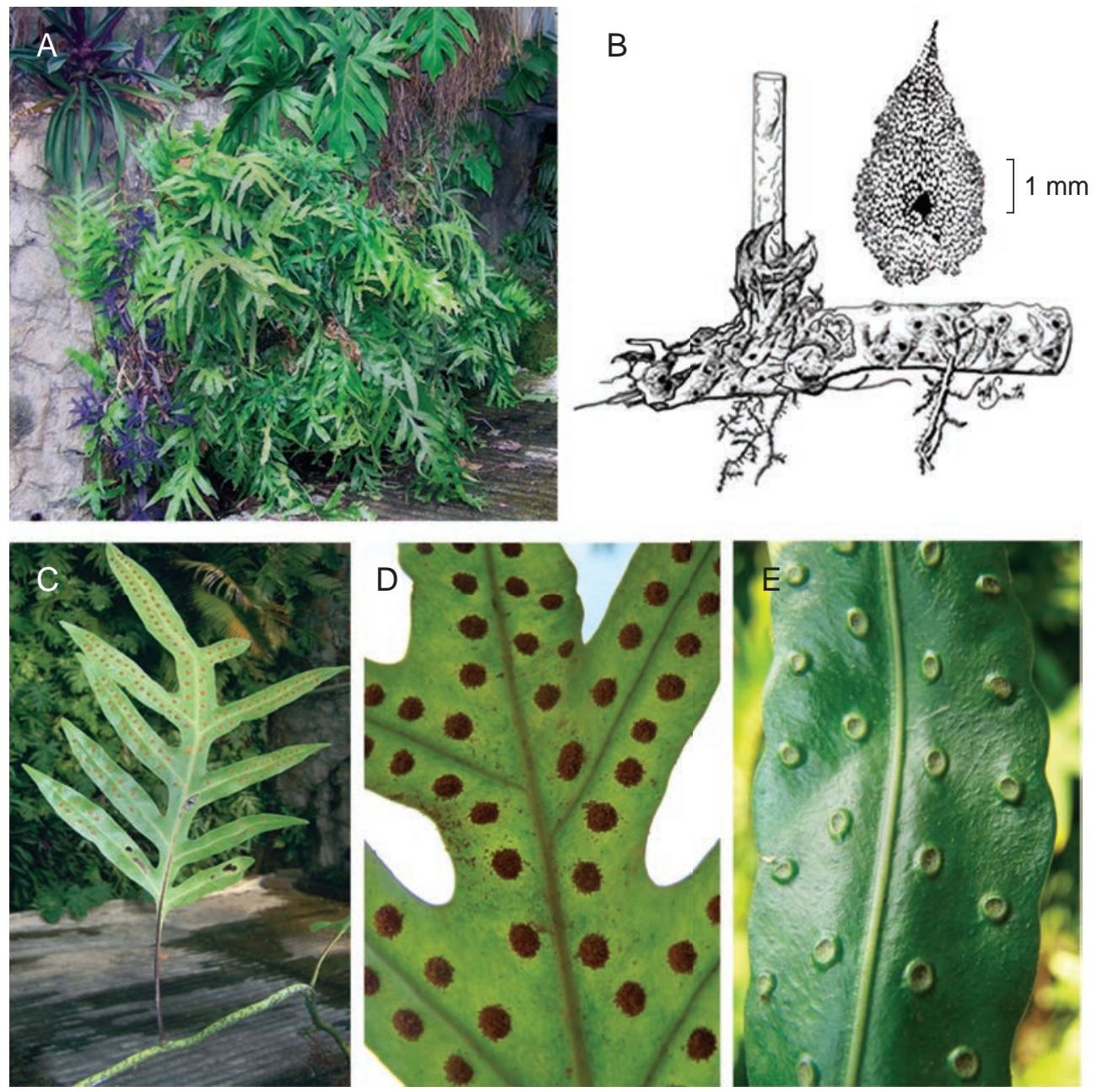

Fig. 1. Phymatosorus grossus. A. Hábitat sobre un muro de contención húmedo en la zona urbana; B. Rizoma y escama (escala $1 \mathrm{~mm}$ ) (dibujo modificado de W.A. Smith, Queensland Herbarium); C. Hábito; D. Vista abaxial de la lámina; E. Vista adaxial de la lámina.

Polinesia Francesas, (LE) n.v. (fide E.A.C.L.E. Schelpe, Contr. Bolus Herb. 1: 100 (1969)). ISOTIPO Langsdorff \& Fischer s.n. (BM !). [Polypodium phymatodes auct. non L.: F.M. Bailey, Lithogr. Ferns Queensland 158 (1892); F.M. Bailey, Queensland Fl. 1985 (1902); F.M. Bailey, Compr. Cat. Queensland Pl. 648 (1913); K. Domin, Biblioth. Bot. 20(85): 177 (1913)]. [Polypodium scolopendria auct. non Burm.f.: Anon., N. Queensland Naturalists Publ. No. 3: 10 (1946)]. [Microsorum scolopendria auct. 
non (Burm.f.) Copel.: D.L. Jones \& S.C. Clemesha, Austral. Ferns \& Fern Allies 224 (1976)].

Phymatodes grossa (Langsdorff \& Fischer) C. Presl, Tent. Pterid. 196. 1836.

Drynaria grossa (Langsdorff \& Fischer) Fée, Mém. Foug. 5: 270. 1852.

Rizoma largamente rastrero, 5-7 mm de diámetro, esclerosado a algo carnoso, glauco, cerradamente escamoso cuando joven y en forma esparcida en la madurez. Escamas del rizoma ovadas, 2.5-8 mm de longitud, 1-3 mm de ancho, adpresas a más o menos cupuladas, deciduas, celdillas clatradas con lúmenes opalescentes y paredes gruesas, de color café obscuro, margen dentado, ápice cortamente acuminado. Hojas maduras (con soros) de 40 a $190 \mathrm{~cm}$ de longitud, apartadas $1-6(-10) \mathrm{cm}$; pecíolo 8-90 cm de largo (1/2 a 3/8 con respecto a la longitud de la hoja), pajizo, glabro excepto algunas escamas en la base; lámina pinnatífida hasta un décimo de su ancho, con 5 a 15 pares de lobos, lobo terminal similar a los laterales (subconformado), raramente simple, de color verde-amarillento, algo coriácea, glabra excepto algunas escamas esparcidas sobre la vena primaria y secundaria (vena media y costa), lobos laterales anchamente separados, largamente lanceolados a oblanceolados, 3-23 cm de longitud, 0.8-4(-5) cm de ancho, ápice agudo u obtuso, base usualmente algo estrechada, margen entero o repando, algo revoluto, vena primaria (central) de la lámina prominente, de color pajizo, acanalada en la superficie superior; vena secundaria, las terciarias y las de orden superior de los segmentos obscurecidas, a contraluz se distinguen areolas primarias dispuestas en varias hileras entre la vena secundaria y el margen, las hileras regulares a irregulares, con vénulas interiores que formar areolas secundarias, última venilla libre. Soros grandes, hasta $5 \mathrm{~mm}$ de diámetro, redondos a elípticos o largamente oblongos (por unión de dos o más soros), dispuestos en (1-)2(-3) hileras irregulares a cada lado de la vena secundaria e incluso en el ala que bordea a la vena primaria entre los lobos, especialmente concentrados en los lobos terminales. Esporas monoletes, 37.5 a $80 \mu \mathrm{m}$ de longitud y 20 a $62.5 \mu \mathrm{m}$ de ancho.

De acuerdo con Nooteboom (1997), esta especie no es diferente de Phymatosorum scolopendria (Burm. f.) Pic. Serm. (= Microsorum scolopendria (Burm.f.) Copel.; nombre con el cual se le identifica ampliamente en la jardinería y comunicaciones científicas del siglo pasado). Sin embargo, según Bostock y Spokes (1998) Phymatosorus grossus se distingue de la anterior por ser plantas fundamentalmente terrestres (vs. mayormente epífitas), tener mayor número de segmentos (5 a 15 pares vs. 1 a 5), rizoma robusto con sus escamas más o menos cupuliformes 
y anchamente ovadas (vs. planas y estrechamente triangulares). P. scolopendria tiene una distribución semejante a la de $P$. grossus, pero sus poblaciones son restringidas y aparentemente no se reporta como invasora o escapada de cultivo en otros países.

Distribución: En forma natural, P. grossus prospera en el norte y noreste de Australia, en Nueva Guinea, Nueva Caledonia, Islas Fidji (Fiji) y a lo largo de las islas del Pacífico sur hasta las islas de la Polinesia Francesa. El conocimiento de su área total es incierto debido a la confusión con la especie hermana Phymatosorum scolopendria, pero es probable que se encuentre también en el sureste asiático y África tropical (Bostock y Spokes, 1998).

En el Nuevo Mundo se le reporta de Florida y principalmente de la zona de las Antillas: Bermudas, Puerto Rico, así como Trinidad y Tobago. En el océano Pacífico fue introducida a Hawai, EUA.

MÉXICO: Tamaulipas, Municipio Gómez Farías, 2303'03" N y 9909'22" O, en jardineras y como trepadora en árboles de la zona urbana y periurbana de Gómez Farías, 380 m s.n.m., 9-VIII-2000, Tejero-Díez 4157 (IZTA, UAMIZ). Guerrero, Municipio Acapulco, calle Anáhuac arriba (al norte) del club de golf y Centro de Convenciones, 1651'32" N y 9951'06" O, jardineras y bardas de contención húmedas, 100 m s.n.m., 27-XII-2010, D. Tejero-Díez 6436a (IZTA, MEXU y ejemplares a repartir); Av. Adolfo López Mateos, entre playa Angosta (Langosta) y La Quebrada, $16^{\circ} 50^{\prime} 41^{\prime \prime} \mathrm{N}$ y $99^{\circ} 54^{\prime} 55^{\prime \prime} \mathrm{O}$, epipétrica en taludes viarios húmedos, 50 m s.n.m., 29XII-2010, D. Tejero-Díez $6436 b$ (IZTA).

Hábitat en México: Es un caméfito (rupícola) a hemicriptófito rizomatoso y trepador a epífito ocasional, siempreverde, termófilo, colonial. Se observa creciendo agresivamente y sin control en macetas, jardineras y jardines. En el medio natural urbano y periurbano se encuentra como epipétrico ruderal y viario, se asocia a escurrimientos de agua permanentes, en sitios expuestos y semiexpuestos al sol. Las poblaciones encontradas en Guerrero distan entre sí cerca de $7 \mathrm{~km} \mathrm{y}$, media entre ellas, la zona marina de la bahía; para explicar esta separación, se puede aducir a la dispersión a media distancia por viento o bien por poblaciones intermedias existentes en las sierras que limitan la bahía de Acapulco.

Comentarios: En la arquitectura de la hoja, Phymatosorus grossus es similar a Phlebodium pseudoaureum (Cav.) Lellinger y a Polypodium eatonii Baker; sin embargo, se distingue de ellas por la presencia de soros impresos en la superficie 
adaxial y el peculiar patrón de venación. En la posición e impresión del soro en la lámina, Phymatosorus grossus se asemeja a Phlebodium inaequale T. Moore, de la cual se distingue por su lámina pinnatífida (vs. completamente pinnada). En la forma de crecimiento, tipo de rizoma y disposición y tipo de las escamas y hojas, Phymatosorus grossus es semejante a Serpocaulon falcaria (Kunze) A.R. Sm., de la cual difiere porque la última especie presenta una hoja pinnatisecta con los segmentos falcados y estrechos, con una venación de una sola hilera de areolas pentagonales a cada lado de la costa.

Usos: Es una especie que tiene un amplio espectro de utilización en los países donde crece naturalmente. Baltrushes (2006) refiere que en las islas Polinesias, se emplea en cinco de los siete grupos de enfermedades registradas en las mujeres, tales como la amenorrea y la falta de menstruación. Se le asocia también al tratamiento de enfermedades microbianas de origen estomacal, tales como las diarreas (Mannar et al., 2008). Se emplea para el control de insectos, sobre todo contra "chinches de cama", en el tratamiento de la esterilidad y para la limpieza del cuerpo en niños y adultos de varios agentes nocivos (Petard, 1972; Whistler, 1992). Cambie y Ash (1994) han identificado varios triterpenoides y esteroles en esta especie. En América es ampliamente vendido como planta ornamental de maceta.

Un reto importante en la investigación de plantas y animales exóticos es la predicción tanto del potencial invasor de una especie, como de los hábitats para ser penetrados (Kareiva, 1996; Vall-Llosera y Sol, 2010). Baker (1974) y Rejmanek y Richardson (1996) han identificado algunos de los atributos de una planta ideal invasora: el rápido crecimiento vegetativo, la producción de una elevada cantidad de propágulos de tamaño pequeño, ya sea por origen sexual o vegetativo y la carencia de un sistema especializado de entrecruzamiento y germinación. Además, se han propuesto varias generalizaciones de tipo ecológico para predecir la irrupción de una especie exótica, tales como el grado de similitud de los ambientes abióticos como el clima, sustrato y topografía nativas vs. el nuevo destino (Cronk y Fuller, 1995; Crawley et al., 1997; Scott y Panetta, 1993; Reichard y Hamilton, 1997), la plasticidad de la planta (carácter de pionera) y el grado de deterioro del nuevo hábitat (Williamson y Fitter, 1996), la carencia de depredadores o parásitos naturales de la especie invasora en su nuevo hábitat (Blossey y Notzold, 1995; Crawley et al., 1997; Strong et al., 1984) o, combinaciones de atributos particulares de las especies exóticas invasoras y ecológicos del ambiente a invadir (Lake y Leishman, 2004). 
Phymatosorus grossus presenta la mayoría de las propiedades descritas anteriormente: Patra y Bera (2007) reportan que esta especie (como P. scolopendria) presenta muy bajo consumo por parte de los herbívoros, inclusive mucho menor que el de otros helechos. La especie tiene una rápida multiplicación vegetativa por medio de sus rizomas y una elevada producción de esporas que tienen potencial de dispersión a larga distancia (Felicísimo y Muñoz, 2010). Produce gametófitos bisexuales con alta probabilidad autogámica (de entrecruzamiento) (Chiou et al., 2002, referida como P. scolopendria). Tiene una amplia plasticidad fenotípica; su hoja varía desde $40 \mathrm{~cm}$ hasta casi $2 \mathrm{~m}$ y puede tener diferentes tonos de coloración de acuerdo con la exposición a la luz. Presenta potencial para ocupar diversos hábitats; fundamentalmente suelos saturados de agua, humícolas e incluso como epipétrico en taludes rocosos. También se ha registrado como epífito trepador. Esta especie se ha convertido en una planta común invasora en Hawai (Wilson, 1996) y con rápida expansión en Florida (Global Invasive Species Database, Anónimo, 2010).

En México, en los sitios de clima cálido y cercanos a las costas, podría competir fácilmente con Adiantum spp., Phlebodium spp., Pityrogramma calomelanos (L.) Link, Serpocaulon falcaria (Kunze) A.R. Sm. y Serpocaulon triseriale (Sw.) A.R. Sm., especie considerada como "amenazada" en la NOM-059 (Anónimo, 2010) en taludes viarios y bosques abiertos. Probablemente pueda crecer también en sitios con suelos saturados con agua.

Con el hallazgo de P. grossus, para México se registran 11 especies de helechos y licopodios introducidos (Cuadro 1) (Mickel y Smith, 2004; Mora-Olivo y Yatskievych, 2009). Sin embargo, probablemente otras 11 especies (Cuadro 2), aprovechando su capacidad de dispersión a larga distancia (Didham et al., 2005; Barbault y Teyssedre, 2010), su carácter de malezas viarias y la gran cantidad de caminos existente en México, han arribado al país recientemente; esta suposición se basa en el hecho de que no se cuenta con registros en los herbarios antes de 1960, sus poblaciones son restringidas en México y disyuntas con respecto a los sitios donde presentan mayor abundancia. Las 22 entidades representan $0.92 \%$ de los pteridobiontes del país, porcentaje muy bajo en comparación con $16 \%$ en el caso de Hawai (Wagner, 1995). En México, la mayoría de estas especies se han naturalizado y forman parte de la vegetación natural donde crecen, a excepción de Nephrolepis cordifolia, N. hirsutula, Phymatossorus grossus, Salvinia molesta y Thelypteris dentata que tienen potencial invasor al desplazar a especies autóctonas y cubrir agresivamente el espacio donde de encuentran. 
Cuadro 1. Especies de pteridobiontes registradas como exóticas en México ( ${ }^{1}$ Mickel y Smith, 2004, ${ }^{2}$ Mora-Olivo y Yatskievych, 2009).

\begin{tabular}{|c|c|c|c|}
\hline Especie & Origen & Comportamiento & $\begin{array}{l}\text { Distribución en México } \\
\text { (Mickel y Smith, 2004) }\end{array}$ \\
\hline $\begin{array}{l}{ }^{1} \text { Adiantum raddianum } \\
\text { C. Presl }\end{array}$ & $\begin{array}{l}\text { Sudamérica, } \\
\text { probablemente } \\
\text { zona nor-andina }\end{array}$ & $\begin{array}{l}\text { Naturalizada, } \\
\text { hierba del } \\
\text { sotobosque }\end{array}$ & $\begin{array}{l}\text { Chis., Mich., Mor., Oax., } \\
\text { Pue., Sin., Tlax., Ver. }\end{array}$ \\
\hline $\begin{array}{l}{ }^{1} \text { Macrothelypteris } \\
\text { torresiana (Gaudich.) } \\
\text { Ching }\end{array}$ & $\begin{array}{l}\text { Asia e islas del } \\
\text { Pacífico }\end{array}$ & Naturalizada & $\begin{array}{l}\text { Chis., Gro., Hgo., Méx., } \\
\text { Mor., Oax., Pue. (Tejero- } \\
\text { Díez 6137, 6149, } 6152 \\
\text { MEXU), Qro., Tab., Ver. }\end{array}$ \\
\hline $\begin{array}{l}{ }^{1} \text { Nephrolepis } \\
\text { cordifolia (L.) C. } \\
\text { Presl }\end{array}$ & Sudamérica & Invasora & $\begin{array}{l}\text { Chis., D.F., Méx., Oax., } \\
\text { Pue., Ver. }\end{array}$ \\
\hline $\begin{array}{l}{ }^{1} \text { Nephrolepis exaltata } \\
\text { (L.) Schott }\end{array}$ & $\begin{array}{l}\text { Centro a } \\
\text { Sudamérica }\end{array}$ & Naturalizada & Ver. \\
\hline $\begin{array}{l}{ }^{1} \text { Nephrolepis falcata } \\
\text { (Cav.) C. Chr. }\end{array}$ & $\begin{array}{l}\text { Malasia y } \\
\text { Polinesia }\end{array}$ & Urbanizada & Gro., Ver. \\
\hline $\begin{array}{l}{ }^{1} \text { Nephrolepis hirsutula } \\
\text { (G. Forst.) C. Presl }\end{array}$ & Centroamérica & Invasora & $\begin{array}{l}\text { Chis., Oax., Pue. (Tejero- } \\
\text { Díez 6139, } 6153 \text { MEXU), } \\
\text { Qro., Tab., Ver., Yuc. }\end{array}$ \\
\hline $\begin{array}{l}\text { Phymatosorus grossus } \\
\text { (Langsd. \& Fisch.) } \\
\text { Brownlie }\end{array}$ & Asia y Oceanía & Urbanizada & Gro., Tamps. \\
\hline${ }^{1}$ Pteris cretica L. & $\begin{array}{l}\text { Asia-África } \\
\text { (incierto) }\end{array}$ & $\begin{array}{l}\text { Naturalizada, } \\
\text { ripícola }\end{array}$ & $\begin{array}{l}\text { Chih., Chis., D.F., Dgo., } \\
\text { Gro., Gto., Hgo., Jal., } \\
\text { Méx., Mich., Mor., N.L., } \\
\text { Oax., Pue., Qro., Sin., } \\
\text { S.L.P., Son., Tam., Ver. }\end{array}$ \\
\hline${ }^{1}$ Pteris vittata $\mathrm{L}$. & Este de Asia & Naturalizada & $\begin{array}{l}\text { N.L., Hgo. (Zuñiga- } \\
\text { Salavatierra } 106 \text { UAEH), } \\
\text { Tam. }\end{array}$ \\
\hline $\begin{array}{l}\text { Salvinia molesta D. } \\
\text { S. Mitchell }\end{array}$ & Sudamérica & $\begin{array}{l}\text { Invasora } \\
\text { hidrófita }\end{array}$ & B.C.N., Son. ${ }^{1}$ \\
\hline $\begin{array}{l}{ }^{1} \text { Thelypteris dentata } \\
\text { (Forssk.) E. P. St. } \\
\text { John }\end{array}$ & $\begin{array}{l}\text { África e Islas } \\
\text { del Pacífico }\end{array}$ & Invasora & $\begin{array}{l}\text { Cam., Chis., Col., Gro., } \\
\text { Mor., Oax., Pue., Qro., } \\
\text { Q.R., Tab., Ver., Yuc. }\end{array}$ \\
\hline
\end{tabular}


Cuadro 2. Especies que probablemente han arribado recientemente a México.

\begin{tabular}{|c|c|c|c|}
\hline Especie & $\begin{array}{l}\text { Sitio con mayor } \\
\text { densidad de } \\
\text { poblaciones }\end{array}$ & Comportamiento & $\begin{array}{l}\text { Distribución en } \\
\text { México (Mickel y } \\
\text { Smith, 2004) }\end{array}$ \\
\hline $\begin{array}{l}\text { Blechnum penna-marina } \\
\text { (Poir.) Kuhn }\end{array}$ & $\begin{array}{l}\text { Sudamérica e islas } \\
\text { circunsubantárticas }\end{array}$ & $\begin{array}{l}\text { Naturalizada, } \\
\text { orillas de } \\
\text { arroyos }\end{array}$ & Chih., Dgo. \\
\hline $\begin{array}{l}\text { Ceratopteris } \\
\text { thalictroides (L.) } \\
\text { Brongn. }\end{array}$ & Asia & $\begin{array}{l}\text { Naturalizada, } \\
\text { hidrófita }\end{array}$ & Gro., Oax. \\
\hline $\begin{array}{l}\text { Equisetum xschaffneri } \\
\text { Milde }\end{array}$ & $\begin{array}{l}\text { Centro a } \\
\text { Sudamérica }\end{array}$ & $\begin{array}{l}\text { Naturalizada, } \\
\text { ripícola }\end{array}$ & $\begin{array}{l}\text { Chis., Oax., Pue., } \\
\text { Tamps., Ver. }^{2}\end{array}$ \\
\hline $\begin{array}{l}\text { Lycopodiella } \\
\text { alopecuroides (L.) } \\
\text { Cranfill }\end{array}$ & Sudamérica & $\begin{array}{l}\text { Naturalizada, } \\
\text { viaria }\end{array}$ & $\begin{array}{l}\text { Chis., Pue. (DTD } \\
\text { 5844, } 5867 \text { IZTA) }\end{array}$ \\
\hline $\begin{array}{l}\text { Lycopodiella caroliniana } \\
\text { (L.) Pic. Serm. }\end{array}$ & Sudamérica & Naturalizada & Tab. \\
\hline $\begin{array}{l}\text { Nephrolepis pectinata } \\
\text { (Willd.) Schott }\end{array}$ & Sudamérica & Invasora & $\begin{array}{l}\text { Chis., Oax., Pue., } \\
\text { Ver. }\end{array}$ \\
\hline $\begin{array}{l}\text { Nephrolepis pendula } \\
\text { (Raddi) J. Sm. }\end{array}$ & Sudamérica & Naturalizada & $\begin{array}{l}\text { Chis., Oax., Tab., } \\
\text { Ver. }\end{array}$ \\
\hline Pteris quadriaurita Retz. & $\begin{array}{l}\text { Australia, Nueva } \\
\text { Zelanda y Polinesia }\end{array}$ & Naturalizada & $\begin{array}{l}\text { Chis., Col., Gro., } \\
\text { Hgo., Jal., Mex., } \\
\text { Mich., Mor., Nay., } \\
\text { Oax., Qro., S.L.P., } \\
\text { Tamps., Ver. }\end{array}$ \\
\hline $\begin{array}{l}\text { Pteris propinqua J. } \\
\text { Agardh }\end{array}$ & Sudamérica & Naturalizada & Ver. \\
\hline Pteris tripartita Sw. & Australia, Polinesia & Naturalizada & Ver. \\
\hline $\begin{array}{l}\text { Thelypteris interrupta } \\
\text { (Willd.) K. Iwats. }\end{array}$ & Asia & Naturalizada & $\begin{array}{l}\text { Camp., Chis., } \\
\text { Hgo., Jal., Q.R., } \\
\text { Tab., Ver. }\end{array}$ \\
\hline
\end{tabular}




\section{AGRADECIMIENTOS}

Robbin Moran y Alan R. Smith auxiliaron en la determinación de los ejemplares mediante el análisis de fotografías. Ellos, J. Canek Ledesma Corral, Leticia Pacheco y George Yatskievych revisaron los herbarios MEXU, MO, NY, UAMIZ y UC respectivamente. Silvia Aguilar Rodríguez, Klaus Mehltreter y Alan R. Smith revisaron críticamente el manuscrito. A los curadores de los herbarios mencionados y los de ENCB y FCMEX se agradece por permitir el acceso a las colecciones.

\section{LITERATURA CITADA}

Adair, R. y R. Groves. 1998. Impact of environmental weeds on biodiversity: a review and development of a methodology. The Director of the National Parks and Wildlife y Biodiversity Group, Environment Australia (formerly Australian Nature Conservation Agency). Canberra, Australia. 55 pp.

Anónimo. 2005. Norma Oficial Mexicana NOM-059-SEMARNAT-2010. Protección ambiental- especies nativas de México de flora y fauna silvestres- categorías de riesgos y especificaciones para su inclusión, exclusión o cambio- lista de especies en riesgo. Diario Oficial de la Federación 2a, sección, 30 de diciembre del 2010. México, D.F. pp. 1-77. http://www.profepa.gob.mx/innovaportal/file/435/1/NOM_059_ SEMARNAT_2010.pdf

Anónimo. 2010. Global invasive species database (GISD). Invasive Species Specialist Group (ISSG)-International Union for Conservation of Nature (IUCN) Species Survival Commission. Consultado el 24 de marzo de 2011. http://www.issg.org/database/ species/ecology.asp?si $=1795 \& \mathrm{fr}=1 \&$ sts=sss\&lang $=\mathrm{EN}$

Baker, H. 1974. The evolution of weeds. Ann. Rev. Ecol. Syst. 5: 1-24.

Baltrushes, N. 2006. Medical ethnobotany, phytochemistry and bioactivity of the ferns of Moorea, French Polynesia. Senior Honors Thesis. Department of Integrative Biology, University of California. Berkeley, California, USA. 46 pp.

Barbault, R. y A. Teyssedre. 2010. Especies invasoras. Investigación y Ciencia: Temas 61: 5258.

Baskin, Y. 2002. The greening of horticulture: new codes of conduct aim to curb plant invasions. BioScience 52(6): 464-471.

Blossey, B. y R. Notzold. 1995. Evolution of increased competitive ability in invasive nonindigenous plants: a hypothesis. J. Ecol. 83: 887-889.

Bosman, M. T. M. 1991. A monograph of the fern genus Microsorum (Polypodiaceae), including an attempt towards a reconstruction of the phylogenetic history of the microsoroids. Leiden Bot. Ser. 14: 1-161. 
Bostock, P. D. y T. M. Spokes. 1998. Polypodiaceae. In: McCarthy, P. M. (ed.). Flora of Australia. Vol. 48. Disponible en Australian Biological Resources Study (ABRS). Consultado el 22 de febrero de 2011. http://www.anbg.gov.au/abrs/online-resources/ flora/redirect.jsp

Cambie, R. C. y J. Ash. 1994. Fijian medicinal plants. The Commonwealth Scientific and Industrial Research Organisation (CSIRO). Campbell, Australia. 365 pp.

Chiou, W. L., D. R. Farrar y T. A. Ranker. 2002. The mating systems of some epiphytic Polypodiaceae. Amer. Fern J. 92(2): 65-79.

Crawley, M., P. Harvey y A. Purvis. 1997. Comparative ecology of the native and alien floras of the British Isles. In: Silvertown, J., M. Franco y J. Harper (eds.). Plant life histories. Ecology, phylogeny and evolution. Cambridge University Press. New York, USA. pp. 36-53.

Cronk, Q. y J. Fuller. 1995. Plant invaders. The threat to natural ecosystems. Chapman and Hall. London, UK. 241 pp.

D’Antonio, C. M. y P. M. Vitousek. 1992. Biological invasions by exotic grasses, the grass/ fire cycle, and global change. Ann. Rev. Ecol. Syst. 23: 63-87.

Didham, R. K., J. M. Tylianakis, M. A. Hutchison, R. M., Ewers y N. J. Gemmell. 2005. Are invasive species the drivers of ecological change? Ecol. Evol. 20: 470-474.

Felicísimo, A. M. y J. Muñoz. 2010. El viento y la dispersión de las plantas (criptógamas continentales). Invest. Cienc. 406: 62-69.

Groves, R. 1991. A short history of biological invasions of Australia. In: Groves, R. y F. Di Castri (eds.). Biogeography of mediterranean invasions. Cambridge University Press. Cambridge, UK. pp. 59-63.

Kareiva, P. 1996. Developing a predictive ecology for non-indigenous species and ecological invasions. Ecology 7: 1651-1652.

Lake, J. C. y M. R. Leishman. 2004. Invasion success of exotic plants in natural ecosystems: the role of disturbance, plant attributes and freedom from herbivores. Biol. Cons. 117: 215-226.

Levine, J. M., M. Vilà, C. M. D’Antonio, J. S. Dukes, K. Grigulis y S. Lavorel. 2003. Mechanisms underlying the impacts of exotic plant invasions. Proc. R. Soc. Lond. (Biol.) 270: 775-781.

Lodge, D. 1993. Biological invasions: lessons from ecology. Trends Ecol. Evol. 8: 133-137.

MacDonald, I. A. W., L. L. Loope, M. B. Usher y O. Hamann. 1989. Wildlife conservation and the invasion of nature reserves by introduced species: a global perspective. In: Drake, J., H. A. Mooney, F. di Castri, R. H. Groves, F. J. Kruger, M. Rejmánek y M. Williamson (eds.). Biological Invasions: a global perspective. John Wiley \& Sons. Chichester, U.K. pp. 215-255.

Mack, R. N., D. Simberloff, W. M. Lonsdale, H. Evans, M. Clout y F. A. Bazzaz. 2000. Biotic invasions: Causes, epidemiology, global consequences and control. Ecol. Appl. 10: 689-710.

Mack, R. N. y M. Erneberg. 2002. The United States naturalized flora: largely the product of deliberate introductions. Ann. Mo. Bot. Gard. 89: 176-189.

Mannar, M. M., M. Maridass y B. Victor. 2008. A review on the potential uses of ferns. Ethnobot. Leaf. 12: 281-285. 
Tejero-Díez y Torres-Díaz: Phymatosorus grossus en México y otros pteridobiontes no-nativos

Mickel, J. T. y A. R. Smith. 2004. The pteridophytes of Mexico. Mem. New York Bot. Gard. 88: 1-1054 (328 láminas).

Mora-Olivo, A. y G. Yatskievych. 2009. Salvinia molesta in Mexico. Am. Fern J. 99(1): 56-58.

Morse, L. E., J. T. Kartesz y L. S. Kutner. 1995. Native vascular plants. In: LaRoe, E. T., G. S. Farris, C. E. Puckett, P. D. Doran y M. J. Mac (eds.). Our living resources: a report to the nation on the distribution, abundance, and health of U.S. plants, animals and ecosystems. U.S. Department of the Interior, National Biological Service. Washington, DC, USA. pp. 205-209.

Nooteboom, H. P. 1997. The microsoroid ferns (Polypodiaceae). Blumea 42: 261-395.

Patra, B. y S. Bera. 2007. Herbivore damage to ferns caused by a chrysomelid beetle from lower gangetic plains of West Bengala, India. Am. Fern J. 97(1): 19-29.

Petard, P. 1972. Raau Tahiti: the use of Polynesia medicinal plants in Tahitian medicine. Technical Paper No. 167. South Pacific Commission. Noumea, New Caledonia. 66 pp.

Pimentel, D. 2005. Environmental and economic costs of the application of pesticides primarily in the United States. Environ. Dev. Sustain. 7: 229-252.

Pysek, P., D. M. Richardson, M. Rejmánek, G. Webster, M. Williamson y J. Kirschner, 2004. Alien plants in checklists and floras: towards a better communication between taxonomists and ecologists. Taxon 53: 131-143.

Reichard, S. H. y C. W. Hamilton. 1997. Predicting invasions of woody plants introduced into North America. Conserv. Biol. 11: 193-203.

Reichard S. H. y P. White. 2001. Horticulture as a pathway of invasive plant introductions in the United States. Biosci. 51: 103-113.

Rejmanek, M. y D. M. Richardson. 1996. What attributes make some plant species more invasive? Ecology 77(6): 1655-1661.

Rose, S. y P. G. Fairweather. 1997. Changes in floristic composition of urban bushland invaded by Pittosporum undulatum in Northern Sydney, Australia. Aust. J. Bot. 45: 123-149.

Scott, J. y F. Panetta. 1993. Predicting the Australian weed status of southern African plants. J. Biogeogr. 20: 87-93.

Smith, A. R., K. M. Pryer, E. Schuettpelz, P. Korall, H. Schneider y P. G. Wolf. 2008. Fern classification. Chapter 16. In: Ranker, T. A. y C. H. Haufler (eds.). The biology and evolution of ferns and lycophytes. Cambridge University Press. New York, USA. pp. 417-467.

Strong, D., J. Lawton y R. Southwood. 1984. Insects on plants: community patterns and mechanisms. Blackwell Scientific. Oxford, UK. 313 pp.

Stuart, T. 2009. Polypods exposed. Fiddlehed forum (Bull. Amer. Fern Soc.) 36(2-3): 9-25.

Tindale, M. D. 1994. Phymatosorus In: McCarthy, P. M. (ed.). Flora of Australia. Vol. 48. Disponible en: ABRS (Australian Biological Resources Study). Consultado el 21 de febrero de 2011. http://www.environment.gov.au/biodiversity/abrs/online-resources/ flora/main/index.html

Vall-Llosera, M. y D. Sol. 2010. Predecir el éxito de las especies invasoras. Investigación y Ciencia 405: 14-15. 
Villaseñor, J. L. y F. J. Espinosa-García. 2004. The alien flowering plants of Mexico. Diversity and Distributions 10: 113-123.

Wagner, W. H. Jr. 1995. Evolution of Hawaiian ferns and fern allies in relation to their conservation status. Pacific Science 49(1): 31-41.

Whistler, W. A. 1992. Botanical inventory of the proposed Ta'u unit of the National Park of American Samoa. Technical Report 83. Botany Department, University of Hawai’i y National Tropical Botanical Garden Lawai, Kaua’i, Hawai’i. Hawaii, USA. 103 pp.

Wilson, K, A. 1996. Alien ferns in Hawaii. Pacific Science 50(2): 127-141.

Williamson, M. y A. Fitter. 1996. The characters of successful invaders. Biol. Conserv. 78: 163-170. 